\title{
A Gauss-Poisson Correspondence and the Lévy Laplacian
}

\author{
Kimiaki SAITÔ \\ Department of Mathematics, Meijo University, Shiogamaguchi 1-501, Tenpaku, Nagoya 468-8502, Japan
}

Received March 9, 2009; final version accepted May 27, 2009

\begin{abstract}
In this paper we present recent results on infinite dimensional Laplacians. In particular, by introducing the operator which transfers regular white noise functionals to functionals of exponential white noise, we give a relationship between an infinite dimensional Fourier-Mehler transform and the Lévy Laplacian. The operator implies a Gauss-Poisson correspondence if we consider the Lévy Laplacian acting on multiple Wiener integrals by some Lévy process. We also give an infinite dimensional random field associated with the Lévy Laplacian.
\end{abstract}

KEYWORDS: A Gauss-Poisson Correspondence, the Lévy Laplacian, an infinite dimensional Fourier-Mehler transform

\section{Introduction}

An infinite dimensional Laplacian was introduced by P. Lévy in his famous book [18]. T. Hida [8] introduced the Laplacian to his theory. This Laplacian has been studied by many authors from various aspects in [1]-[4], [6], [7], [11], [12], [16], [17], [19], [21], [23] and [27], and references cited therein. On the other hand an infinite dimensional Fourier-Mehler transform was introduced by H.-H. Kuo in [14]. This transform has been also studied by many authors (see [5], [9], [15], [26] and others).

A relationship between the Lévy Laplacian $\Delta_{L}$ and the number operator is discussed in our previous paper [27] by introducing some operator which transfers Gaussian white noise functionals to exponential functionals of Gaussian white noise. However any isomorphism between those functionals is not discussed in the paper.

The purposes of this paper are to construct an infinite dimensional random field associated with the Lévy Laplacian and also to give relationships between the Lévy Laplacian, number operator and an infinite dimensional Fourier-Mehler transform $\mathcal{F}_{\theta}, \theta \in \mathbf{R}$, by an operator $K$ which plays the role of a Gauss-Poisson correspondence given as an isomorphism from a space of Gaussian noise functionals onto a space of Poisson noise functionals (see Section 5).

The relationship is given by

$$
K\left[\mathcal{F}_{\theta} \varphi\right]=e^{-i \theta|T| \Delta_{L}} K \varphi \diamond e^{-i \frac{\theta}{2}|T| \Delta_{L}} K g \diamond e^{-i \frac{\pi}{4}|T| \Delta_{L}} K g,
$$

for all $\varphi$ in some domain of Gaussian noise functionals, where $|T|$ is the length of an interval $T$ and $\diamond$ means the Wick product (Proposition 5.4). This means that $K\left[\mathcal{F}_{\theta} \varphi\right]$ satisfies the equation:

$$
\frac{\partial K\left[\mathcal{F}_{\theta} \varphi\right]}{\partial \theta}=-i|T| \Delta_{L} K\left[\mathcal{F}_{\theta} \varphi\right]+i\left(1-\frac{1}{2} e^{2 i \theta}\right) K\left[\int_{T}: x(t)^{2}: d t\right] \diamond K\left[\mathcal{F}_{\theta} \varphi\right]
$$

where $\int_{T}: x(t)^{2}: d t$ is a Gaussian noise distribution of which the $S$-transform is given by $S\left[\int_{T}: x(t)^{2}: d t\right](\xi)=$ $\int_{T} \xi(t)^{2} d t, \xi \in E_{\mathbf{C}}$ (Theorem 5.5).

This paper is organized as follows. In Section 2, we summarize basic elements of an infinite dimensional analysis placing a special emphasis on a stochastic process given by a Lévy process as a linear combination of a Brownian motion and a standard Poisson process. In Section 3, extending the recent works in [11] and [28], we formulate the Lévy Laplacian acting on a Hilbert space consisting of some Lévy distributions and give an equi-continuous $C_{0^{-}}$ semigroup generated by the Laplacian. This situation is further generalized in Section 4 and we give an infinite dimensional random field associated with the extended Lévy Laplacian through the semigroup. This is a generalization of results in [28] and [29]. In Section 5, we introduce an isomorphism from a space of Gaussian regular functionals onto a space of Poisson noise functionals. This is a Gauss-Poisson correspondence. The correspondence gives a relationship between the Lévy Laplacian and the number operator. Moreover, we give a relationship between Kuo's Fourier-Mehler transform and the unitary group generated by the Lévy Laplacian through the correspondence.

\section{Functionals of Gaussian noise and Poisson noise}

Let $\{B(t)\}_{t \in \mathbf{R}}$ be a Brownian motion and $\{N(t)\}_{t \in \mathbf{R}}$ a standard Poisson process which is independent of the Brownian motion. For any $\sigma \geq 0$ and $\lambda \in \mathbf{R}$, we set $\Lambda_{\sigma, \lambda}(t)=\sigma B(t)+\lambda N(t), t \geq 0$. Then we have 


$$
\begin{gathered}
E\left[e^{i z \Lambda_{\sigma, \lambda}(t)}\right]=e^{t h(z)}, \quad t \in \mathbf{R}, \\
h(z)=-\frac{\sigma^{2}}{2} z^{2}+e^{i \lambda z}-1 .
\end{gathered}
$$

Let $E=\wp(\mathbf{R})$ be the Schwartz space of rapidly decreasing $\mathbf{R}$-valued functions on $\mathbf{R}$ and let $E^{*}$ be a dual space of $E$. Then there exists an orthonormal basis $\left\{e_{v}\right\}_{v \geq 0}$ of $L^{2}(\mathbf{R})$ contained in $E$ such that $A e_{v}=2(v+1) e_{v}, v=0,1,2, \ldots$, with $A=-\frac{d^{2}}{d u^{2}}+u^{2}+1$. For $p \in \mathbf{R}$ define a norm $|\cdot|_{p}$ by $|f|_{p}=\left|A^{p} f\right|_{L^{2}(\mathbf{R})}$ for $f \in E$ and let $E_{p}$ be the completion of $E$ with respect to the norm $|\cdot|_{p}$. The canonical bilinear form on $E^{*} \times E$ is denoted by $\langle\cdot, \cdot\rangle$. We denote the complexifications of $L^{2}(\mathbf{R}), E$ and $E_{p}$ by $L_{\mathbf{C}}^{2}(\mathbf{R}), E_{\mathbf{C}}$ and $E_{\mathbf{C}, p}$, respectively.

Set

$$
C(\xi)=\exp \left\{\int_{\mathbf{R}} h(\xi(u)) d u\right\}, \quad \xi \in E .
$$

Then by the Bochner-Minlos theorem, there exists a probability measure $\mu_{\sigma, \lambda}$ on $E^{*}$ such that

$$
\int_{E^{*}} \exp \{i\langle x, \xi\rangle\} d \mu_{\sigma, \lambda}(x)=C(\xi), \quad \xi \in E .
$$

Let $\left(L^{2}\right)_{\sigma, \lambda} \equiv L^{2}\left(E^{*}, \mu_{\sigma, \lambda}\right)$ be the Hilbert space of $\mathbf{C}$-valued square-integrable functions on $E^{*}$ with $L^{2}$-norm $\|\cdot\|_{\sigma, \lambda}$ with respect to $\mu_{\sigma, \lambda}$. The Wiener-Itô decomposition theorem says that:

$$
\left(L^{2}\right)_{\sigma, \lambda}=\bigoplus_{n=0}^{\infty} H_{n}
$$

where $H_{n}$ is the space of multiple Wiener integrals of order $n \in \mathbf{N}$ and $H_{0}=\mathbf{C}$ by definition. According to (2.2), each $\phi \in\left(L^{2}\right)_{\sigma, \lambda}$ is represented as

$$
\varphi=\sum_{n=0}^{\infty} \mathbf{I}_{n}\left(f_{n}\right), \quad f_{n} \in L_{\mathbf{C}}^{2}(\mathbf{R})^{\hat{\otimes} n},
$$

where $L_{\mathbf{C}}^{2}(\mathbf{R})^{\hat{\otimes} n}$ denotes the $n$-fold symmetric tensor power of $L_{\mathbf{C}}^{2}(\mathbf{R})$ (in the sense of a Hilbert space) and $\mathbf{I}_{n}\left(f_{n}\right)$ is given by

$$
\mathbf{I}_{n}\left(f_{n}\right)=\int_{\mathbf{R}^{n}} f_{n}\left(u_{1}, \ldots, u_{n}\right) d \widetilde{\Lambda_{\sigma, \lambda}}\left(u_{1}\right) \cdots d \widetilde{\Lambda_{\sigma, \lambda}}\left(u_{n}\right)
$$

where $\left\{\widetilde{\Lambda_{\sigma, \lambda}}(t) \equiv \Lambda_{\sigma, \lambda}(t)-\lambda t ; t \in \mathbf{R}\right\}$ is the compensated stochastic process. The $U$-transform of $\varphi \in\left(L^{2}\right)_{\sigma, \lambda}$ is defined by

$$
\mathcal{U}[\varphi](\xi)=\int_{E^{*}} \varphi(x) \varphi_{\xi}(x) d \mu_{\sigma, \lambda}(x), \quad \xi \in E,
$$

where $\varphi_{\xi}(x)=C(\xi)^{-1} \exp (i\langle x, \xi\rangle)$.

For $p \in \mathbf{R}$, let $\|\varphi\|_{p, \sigma, \lambda}=\left\|\Gamma(A)^{p} \varphi\right\|_{\sigma, \lambda}$, where $\Gamma(A)$ is the second quantization operator of $A$ given by

$$
\Gamma(A) \varphi=\sum_{n=0}^{\infty} \mathbf{I}_{n}\left(A^{\otimes n} f_{n}\right)
$$

for $\varphi=\sum_{n=0}^{\infty} \mathbf{I}_{n}\left(f_{n}\right) \in\left(L^{2}\right)_{\sigma, \lambda}$ if (2.3) exists in $\left(L^{2}\right)_{\sigma, \lambda}$. If $p \geq 0$, let $(E)_{p ; \sigma, \lambda}$ be the domain of $\Gamma(A)^{p}$. If $p<0$, let $(E)_{p ; \sigma, \lambda}$ be the completion of $\left(L^{2}\right)_{\sigma, \lambda}$ with respect to the norm $\|\cdot\|_{p, \sigma, \lambda}$. Then $(E)_{p ; \sigma, \lambda}, p \in \mathbf{R}$, is a Hilbert space with the norm $\|\cdot\|_{p, \sigma, \lambda}$. The projective limit space $(E)_{\sigma, \lambda}$ of spaces $(E)_{p ; \sigma, \lambda}, p \in \mathbf{R}$, is a nuclear space. The inductive limit space $(E)_{\sigma, \lambda}^{*}$ of spaces $(E)_{p ; \sigma, \lambda}, p \in \mathbf{R}$, is nothing but the strong dual space of $(E)_{\sigma, \lambda}$.

The $\mathcal{U}$-transform on $\left(L^{2}\right)_{\sigma, \lambda}$ can be extended to an operator defined on $(E)_{\sigma, \lambda}^{*}$ by using the canonical bilinear form $\langle\langle\cdot, \cdot\rangle\rangle_{\sigma, \lambda}$ on $(E)_{\sigma, \lambda}^{*} \times(E)_{\sigma, \lambda}$ as

$$
\mathcal{U}[\Phi](\xi)=\left\langle\left\langle\Phi, \varphi_{\xi}\right\rangle\right\rangle_{\sigma, \lambda}, \quad \xi \in E .
$$

For $\Phi=\sum_{n=0}^{\infty} \mathbf{I}_{n}\left(F_{n}\right) \in(E)_{\sigma, 0}^{*}$ and $\varphi=\sum_{n=0}^{\infty} \mathbf{I}_{n}\left(f_{n}\right) \in(E)_{\sigma, 0}$, we have

$$
\langle\langle\Phi, \varphi\rangle\rangle_{\sigma, 0}=\sum_{n=0}^{\infty} n ! \sigma^{2 n}\left\langle F_{n}, f_{n}\right\rangle .
$$

For $\Phi=\sum_{n=0}^{\infty} \mathbf{I}_{n}\left(F_{n}\right) \in(E)_{0, \lambda}^{*}$ and $\varphi=\sum_{n=0}^{\infty} \mathbf{I}_{n}\left(f_{n}\right) \in(E)_{0, \lambda}$, we have

$$
\langle\langle\Phi, \varphi\rangle\rangle_{0, \lambda}=\sum_{n=0}^{\infty} n ! \lambda^{2 n}\left\langle F_{n}, f_{n}\right\rangle .
$$




\section{The Lévy Laplacian acting on Gaussian and Poisson noise functionals}

Fixing a finite interval $T$ of $\mathbf{R}$, we take an orthonormal basis $\left\{\zeta_{n}\right\}_{n=0}^{\infty} \subset E$ for $L^{2}(T)$ which is equally dense and uniformly bounded:

$$
\frac{1}{N} \sum_{n=0}^{N-1} \zeta_{n}(t)^{2} \rightarrow \frac{1}{|T|} \text { in } L^{2}(T) ; \quad \sup _{n \geq 0} \sup _{t \in T}\left|\zeta_{n}(t)\right|<\infty .
$$

Let $\mathscr{D}_{L}$ denote the set of all $\varphi \in(E)_{\sigma, \lambda}^{*}$ such that the limit

$$
\widetilde{\Delta}_{L}(U \varphi)(\xi)=\lim _{N \rightarrow \infty} \frac{1}{N} \sum_{n=0}^{N-1}(U \varphi)^{\prime \prime}(\xi)\left(\zeta_{n}, \zeta_{n}\right),
$$

exists for any $\xi \in E$ and $\widetilde{\Delta}_{L}(\mathcal{U} \varphi)$ is in $U\left[(E)_{\sigma, \lambda}^{*}\right]$. The Lévy Laplacian $\Delta_{L}$ on $\mathscr{D}_{L}$ is defined by $\Delta_{L} \varphi=\mathcal{U}^{-1} \widetilde{\Delta}_{L} U_{\varphi}$, $\varphi \in \mathscr{D}_{L}$.

For any $\sigma \geq 0, \lambda>0$, and $n \in \mathbf{N}$ let $\mathbf{E}_{\sigma, \lambda, n}$ denote the set of $\varphi \in\left(L^{2}\right)_{\sigma, \lambda}$ given by the form:

$$
\varphi=\int_{T^{n}} f\left(u_{1}, \ldots, u_{n}\right) d \Lambda_{\sigma, \lambda}\left(u_{1}\right) \cdots d \Lambda_{\sigma, \lambda}\left(u_{n}\right),
$$

of which the $U$-transform is given by

$$
\begin{gathered}
\mathcal{U}(\xi)=\int_{T^{n}} f\left(u_{1}, \ldots, u_{k}\right) \prod_{j=1}^{n} \Xi_{\sigma, \lambda}(\xi)\left(u_{j}\right) d u_{1} \ldots d u_{k}, \quad \xi \in E, \\
\Xi_{\sigma, \lambda}(\xi)(u)=i \sigma^{2} \xi(u)+\lambda e^{i \lambda \xi(u)}
\end{gathered}
$$

where $f$ belongs to $L_{\mathbf{C}}^{2}(\mathbf{R})^{\hat{\otimes} n}$ with supp $f \subset T^{n}$.

Set $\mathbf{E}_{\sigma, \lambda, 0}=\mathbf{C}$ for any $\sigma \geq 0$ and $\lambda>0$. Then $\mathbf{E}_{\sigma, \lambda, n}$ is a closed linear subspace of $\left(L^{2}\right)_{\sigma, \lambda}$. By direct computations through the $U$-transform, we have the following.

Theorem 3.1 (cf. [28]). For each $\sigma \geq 0, n \in \mathbf{N}$ and $\lambda>0$, the Lévy Laplacian $\Delta_{L}$ becomes a continuous linear operator on $\mathbf{E}_{\sigma, 0, n} \oplus \mathbf{E}_{0, \lambda, n}$ such that $\Delta_{L} \varphi=0$ for all $\varphi \in \mathbf{E}_{\sigma, 0, n}$ and $\Delta_{L} \varphi=-\frac{n \lambda^{2}}{|T|} \varphi$ for all $\varphi \in \mathbf{E}_{0, \lambda, n}$.

For $N \in \mathbf{N}$ and $\lambda>0$ let $\mathbf{D}_{N}^{0, \lambda}$ be the space of $\varphi \in\left(L^{2}\right)_{0, \lambda}$ which admits an expression $\varphi=\sum_{n=1}^{\infty} \varphi_{n}, \varphi_{n} \in \mathbf{E}_{0, \lambda, n}$, such that $\|\varphi\|\left\|_{N, 0, \lambda}^{2}=\sum_{n=1}^{\infty} \alpha_{N}^{\lambda}(n)\right\| \varphi_{n} \|_{0, \lambda}^{2}<\infty$, where $\alpha_{N}^{\lambda}(n)=\sum_{\ell=0}^{N}\left(\frac{n \lambda^{2}}{|T|}\right)^{2 \ell}$.

By the Schwartz inequality we see that $\mathbf{D}_{N}^{0, \lambda}$ is a subspace of $\left(L^{2}\right)_{0, \lambda}$ and becomes a Hilbert space equipped with the new norm $\||\cdot|\|_{N, 0, \lambda}$. Moreover, in view of the inclusion relations:

$$
\left(L^{2}\right)_{0, \lambda} \supset \mathbf{D}_{1}^{0, \lambda} \supset \cdots \supset \mathbf{D}_{N}^{0, \lambda} \supset \mathbf{D}_{N+1}^{0, \lambda} \supset \cdots,
$$

we define

$$
\mathbf{D}_{\infty}^{0, \lambda}=\underset{N \rightarrow \infty}{\operatorname{proj} \lim } \mathbf{D}_{N}^{0, \lambda}=\bigcap_{N=1}^{\infty} \mathbf{D}_{N}^{0, \lambda} .
$$

Then $\Delta_{L}$ is a self-adjoint operator densely defined in $\mathbf{D}_{N}^{0, \lambda}$ for each $N \in \mathbf{N}$ and $\lambda>0$. In view of the action in Theorem 3.1, for each $z \in \mathbf{C}$ with $\operatorname{Re} z \geq 0$ and $\lambda>0$ we consider an operator $G_{z}^{\lambda}$ on $\mathbf{D}_{\infty}^{0, \lambda}$ defined by

$$
G_{z}^{\lambda} \varphi=\sum_{n=1}^{\infty} e^{-z n \lambda^{2} /|T|} \varphi_{n}, \quad \varphi=\sum_{n=1}^{\infty} \varphi_{n} \in \mathbf{D}_{\infty}^{0, \lambda} .
$$

We also define $G_{z}^{0}$ on $\left(L^{2}\right)_{\sigma, 0}$ as an identity operator $I$ by $I \varphi=\varphi, \varphi \in\left(L^{2}\right)_{\sigma, 0}$.

Theorem 3.2. Let $\lambda>0$. Then the family $\left\{G_{t}^{\lambda} ; t \geq 0\right\}$ on $\mathbf{D}_{\infty}^{0, \lambda}$ is an equi-continuous $C_{0}$-semigroup generated by $\Delta_{L}$.

Proof. With the help of Theorem 3.1, the proof is a modification of that of Proposition 5 in [29].

\section{An extension of the Lévy Laplacian and an associated random field}

Let $d \nu(\lambda)$ be a finite Borel measure on $\mathbf{R}$ satisfying

$$
\int_{(0, \infty)} \frac{d v(\lambda)}{\lambda^{4}}<\infty
$$

Fix $N \in \mathbf{N}$ and $a \geq 0$. Let $\mathfrak{D}_{N}^{\sigma}$ be the space of (equivalent classes of) measurable vector functions $\varphi=\left(\varphi^{\lambda}\right)$ with $\varphi^{\lambda}=\sum_{n=1}^{\infty} \varphi_{n}^{\lambda} \in \overline{\mathbf{D}}_{N}^{0, \lambda}$ for all $\lambda>0$, and $\varphi^{0} \in\left(L^{2}\right)_{\sigma, 0}$, such that 


$$
\left\|\left|\varphi\|\|_{N}^{2}=\left\|\varphi^{0}\right\|_{\sigma, 0}^{2}+\sum_{n=1}^{\infty} \int_{(0, \infty)}\left\|\mid \varphi_{n}^{\lambda}\right\| \|_{N, 0, \lambda}^{2} d \nu(\lambda)<\infty .\right.\right.
$$

Then $\mathfrak{D}_{N}^{\sigma}$ becomes a Hilbert space with the norm given in (4.1).

In view of the natural inclusion $\mathfrak{D}_{N+1}^{\sigma} \subset \mathfrak{D}_{N}^{\sigma}$ for $N \in \mathbf{N}$, which is obvious from construction, we define $\mathfrak{D}_{\infty}^{\sigma}=$ proj $\lim _{N \rightarrow \infty} \mathfrak{D}_{N}^{\sigma}=\bigcap_{N=1}^{\infty} \mathfrak{D}_{N}^{\sigma}$.

The Lévy Laplacian $\Delta_{L}$ is defined on the space $\mathfrak{D}_{\infty}^{\sigma}$ by

$$
\Delta_{L} \boldsymbol{\varphi}=\left(\Delta_{L} \varphi^{\lambda}\right), \quad \boldsymbol{\varphi}=\left(\varphi^{\lambda}\right) \in \mathfrak{D}_{\infty}^{\sigma} .
$$

Then $\Delta_{L}$ is a continuous linear operator from $\mathfrak{D}_{\infty}^{\sigma}$ into itself. Similarly for $z \in \mathbf{C}$ with $\operatorname{Re} z \geq 0$ we define

$$
G_{z} \boldsymbol{\varphi}=\left(G_{z}^{\lambda} \varphi^{\lambda}\right), \quad \boldsymbol{\varphi}=\left(\varphi^{\lambda}\right) \in \mathfrak{D}_{\infty}^{\sigma} .
$$

Then we have the following result by Theorem 3.2 and by a similar method as in the proof of Theorem 3.3 in [24].

Theorem 4.1. The family $\left\{G_{t} ; t \geq 0\right\}$ is an equi-continuous $C_{0}$-semigroup generated by $\Delta_{L}$ as an operator acting on $\mathfrak{D}_{\infty}^{\sigma}$.

For $p \in \mathbf{R}$ let $E_{p}^{[0, \infty)}$ be the linear space of all functions $\lambda \mapsto \xi_{\lambda} \in E_{p}, \lambda \geq 0$, which are strongly measurable. An element of $E_{p}^{[0, \infty)}$ is denoted by $\xi=\left(\xi_{\lambda}\right)_{\lambda \geq 0}$. Equipped with the metric given by

$$
d_{p}(\boldsymbol{\xi}, \boldsymbol{\eta})=\int_{[0, \infty)} \frac{\left|\xi_{\lambda}-\eta_{\lambda}\right|_{p}}{1+\left|\xi_{\lambda}-\eta_{\lambda}\right|_{p}} d \nu(\lambda), \quad \xi=\left(\xi_{\lambda}\right), \quad \eta=\left(\eta_{\lambda}\right),
$$

the space $E_{p}^{[0, \infty)}$ becomes a complete metric space. In view of $d_{p} \leq d_{q}$ for $p \geq q$, we introduce the projective limit space

$$
E^{[0, \infty)}=\underset{p \rightarrow \infty}{\operatorname{proj} \lim } E_{p}^{[0, \infty)}
$$

Similarly, let $\mathbf{C}^{[0, \infty)}$ denote the linear space of all measurable functions $\lambda \mapsto z_{\lambda} \in \mathbf{C}, \lambda \geq 0$, equipped with the metric defined by

$$
\rho(\mathbf{z}, \mathbf{u})=\int_{[0, \infty)} \frac{\left|z_{\lambda}-u_{\lambda}\right|}{1+\left|z_{\lambda}-u_{\lambda}\right|} d \nu(\lambda), \quad \mathbf{z}=\left(z_{\lambda}\right), \quad \mathbf{u}=\left(u_{\lambda}\right) .
$$

Then $\mathbf{C}^{[0, \infty)}$ is also a complete metric space.

The $U$-transform can be extended to a continuous linear operator on $\mathfrak{D}_{\infty}^{\sigma}$ by

$$
\mathcal{U}(\xi)=\left(U \varphi^{\lambda}\left(\xi_{\lambda}\right)\right)_{\lambda \geq 0}, \quad \xi=\left(\xi_{\lambda}\right)_{\lambda \geq 0} \in E^{[0, \infty)},
$$

for any $\boldsymbol{\varphi}=\left(\varphi^{\lambda}\right)_{\lambda \geq 0} \in \mathfrak{D}_{\infty}^{\sigma}$. The space $\mathcal{U}\left[\mathfrak{D}_{\infty}^{\sigma}\right]$ is endowed with the topology induced from $\mathfrak{D}_{\infty}^{\sigma}$ by the $\mathcal{U}$-transform. Then the $\mathcal{U}$-transform becomes a homeomorphism from $\mathfrak{D}_{\infty}^{\sigma}$ onto $\mathcal{U}\left[\mathfrak{D}_{\infty}^{\sigma}\right]$. The transform $\mathcal{U} \boldsymbol{\varphi}$ of $\boldsymbol{\varphi} \in \mathfrak{D}_{\infty}^{\sigma}$ is a continuous operator from $E^{[0, \infty)}$ into $\mathbf{C}^{[0, \infty)}$. We denote the operator by the same notation $U \boldsymbol{\varphi}$.

Let $\left\{X_{t}^{1} ; t \geq 0\right\}$ and $\left\{X_{t}^{2} ; t \in \mathbf{R}\right\}$ be independent stochastic processes, of which the characteristic functions are given by

$$
\mathbf{E}\left[e^{i \alpha X_{t}^{1}}\right]=e^{-t|\alpha|}, \quad \alpha \in \mathbf{R}
$$

and

$$
\mathbf{E}\left[e^{i \alpha X_{t}^{2}}\right]=e^{-i t|\alpha|}, \quad \alpha \in \mathbf{R}
$$

respectively. Take a smooth function $\eta_{T} \in E$ with $\eta_{T}=1 /|T|$ on $T$, and set $Y_{z}^{\lambda}=X_{\lambda \operatorname{Re} z}^{1} \eta_{T}+X_{\lambda \operatorname{Im} z}^{2} \eta_{T}$ for $\lambda \geq 0$ and $z \in \mathbf{C}$ with $\operatorname{Re} z \geq 0$. Define an infinite dimensional random field $\left\{\mathbf{Y}_{z} ; z \in \mathbf{C}, \operatorname{Re} z \geq 0\right\}$ starting at $\boldsymbol{\xi}=\left(\xi_{\lambda}\right)_{\lambda \geq 0} \in E^{[0, \infty)}$ by

$$
\mathbf{Y}_{z}=\left(\xi_{\lambda}+Y_{z}^{\lambda}\right)_{\lambda \geq 0}, \quad \operatorname{Re} z \geq 0
$$

Then this is an $E^{[0, \infty)}$-valued random field and we have the following

Theorem 4.2. If $F$ is the $U$-transform of an element in $\mathfrak{D}_{\infty}^{\sigma}$, we have

$$
\widetilde{G_{z}} F(\xi)=E\left[F\left(\mathbf{Y}_{z}\right) \mid \mathbf{Y}_{0}=\xi\right], \quad \operatorname{Re} z \geq 0
$$

where $\widetilde{G_{z}}=U G_{z} U^{-1}$.

Proof. We first consider the case when $F \in \mathcal{U}\left[\mathfrak{D}_{\infty}^{\sigma}\right]$ is given by

$$
\begin{gathered}
F(\boldsymbol{\xi})=\left(F^{\lambda}\left(\xi_{\lambda}\right)\right)_{\lambda \geq 0}, \quad F^{0} \in \mathcal{U}\left[\left(L^{2}\right)_{\sigma, 0}\right], \\
F^{\lambda}\left(\xi_{\lambda}\right)=\lambda^{n} \int_{T^{n}} f(\mathbf{u}) \cdot \prod_{j=1}^{n} e^{i \lambda \xi_{\lambda}\left(u_{j}\right)} d \mathbf{u},
\end{gathered}
$$


with $f \in L_{\mathbf{C}}^{2}(\mathbf{R})^{\hat{\otimes} n}$. Then we have

$$
\begin{aligned}
& E\left[F\left(\mathbf{Y}_{z}\right) \mid \mathbf{Y}_{0}=\xi\right]=\left(E\left[F^{\lambda}\left(\xi_{\lambda}+Y_{z}^{\lambda}\right)\right]\right)_{\lambda \geq 0} \\
& \quad=\left(F^{0}\left(\xi_{0}\right) \delta_{\lambda, 0}+\lambda^{n} \int_{T^{n}} f(\mathbf{u}) \prod_{j=1}^{n} e^{i \lambda \xi_{\lambda}\left(u_{j}\right)} E\left[e^{i n \frac{\lambda}{T T} Y_{z}^{\lambda}}\right] d \mathbf{u}\right)_{\lambda \geq 0} \\
& \quad=\left(e^{-z n \lambda^{2} /|T|} F^{\lambda}\left(\xi_{\lambda}\right)\right)_{\lambda \geq 0}=\left(\widetilde{G_{z}^{\lambda}} F^{\lambda}\left(\xi_{\lambda}\right)\right)_{\lambda \geq 0}=\widetilde{G_{z}} F(\boldsymbol{\xi}) .
\end{aligned}
$$

Next let $F=\left(F^{0} \delta_{\lambda, 0}+\sum_{n=1}^{\infty} F_{n}^{\lambda}\right)_{\lambda \geq 0} \in \mathcal{U}\left[\mathfrak{D}_{\infty}^{\sigma}\right]$. Then for $v$-almost all $\lambda>0$ and for any $n \in \mathbf{N}, F_{n}^{\lambda}$ is expressed in the following form:

$$
F_{n}^{\lambda}\left(\xi_{\lambda}\right)=\lambda^{n} \int_{T^{n}} f_{\lambda, n}(\mathbf{u}) \prod_{j=1}^{n} e^{i \lambda \xi_{\lambda}\left(u_{j}\right)} d \mathbf{u} .
$$

Since $F^{0} \in \mathcal{U}\left[\left(L^{2}\right)_{\sigma, 0}\right]$ and $F_{n}^{\lambda} \in \mathcal{U}\left[\mathbf{D}_{\infty}^{0, \lambda}\right]$, there exist $\varphi^{0} \in\left(L^{2}\right)_{\sigma, 0}$ and $\varphi_{n}^{\lambda} \in \mathbf{D}_{\infty}^{0, \lambda}$ such that $F^{0}=\mathcal{U}\left[\varphi^{0}\right]$ and $F_{n}^{\lambda}=U\left[\varphi_{n}^{\lambda}\right]$ for $\nu$-almost all $\lambda$ and each $n$. By the Schwarz inequality, we see that

$$
\begin{aligned}
& \sum_{n=1}^{\infty} E\left[\left|F_{n}^{\lambda}\left(\xi_{\lambda}+Y_{z}^{\lambda}\right)\right|\right] \leq \sum_{n=0}^{\infty}\left\|\varphi_{n}^{\lambda}\right\|_{0, \lambda} E\left[\left\|\varphi_{\xi_{\lambda}+Y_{z}^{\lambda}}\right\|_{0, \lambda}\right] \\
& \quad \leq\left\{\sum_{n=1}^{\infty} \alpha_{N}^{\lambda}(n)^{-1}\right\}^{1 / 2}\left\{\sum_{n=1}^{\infty} \alpha_{N}^{\lambda}(n)\left\|\varphi_{n}^{\lambda}\right\|_{0, \lambda}^{2}\right\}^{1 / 2} E\left[\left\|\varphi_{\xi_{\lambda}+Y_{z}^{\lambda}}\right\|_{0, \lambda}\right]<\infty,
\end{aligned}
$$

where $\varphi_{\xi_{\lambda}}=C\left(\xi_{\lambda}\right)^{-1} e^{\left.i l, \xi_{\lambda}\right\rangle}$ for $\nu$-almost all $\lambda \geq 0$ and each $N \in \mathbf{N}$. Therefore by the continuity of $\widetilde{G_{z}^{\lambda}}, \lambda \geq 0$, we get that

$$
\begin{aligned}
E\left[F\left(\boldsymbol{\xi}+\mathbf{Y}_{z}\right)\right] & =\left(\sum_{n=1}^{\infty} E\left[F_{n}^{\lambda}\left(\xi_{\lambda}+Y_{z}^{\lambda}\right)\right]\right)_{\lambda \geq 0}=\left(\sum_{n=1}^{\infty} \widetilde{G_{z}^{\lambda}} F_{n}^{\lambda}\left(\xi_{\lambda}\right)\right)_{\lambda \geq 0} \\
& =\left(\widetilde{G_{z}^{\lambda}} \sum_{n=1}^{\infty} F_{n}^{\lambda}\left(\xi_{\lambda}\right)\right)_{\lambda \geq 0}=\widetilde{G_{z}} F(\xi) .
\end{aligned}
$$

Thus we obtain the assertion.

For any $\eta \in E^{[0, \infty)}$ we define a translation operator $T_{\eta}$ on $\mathfrak{D}_{\infty}^{\sigma}$ by

$$
\left(U T_{\eta} \varphi\right)(\xi)=(U \varphi)(\xi+\eta) .
$$

Then by Theorem 4.2 we have the following stochastic expression of $G_{t}$.

Theorem 4.3. For all $\varphi$ in $\mathfrak{D}_{\infty}^{\sigma}$ we have

$$
G_{z} \varphi=E\left[T_{\left(Y_{z}^{\lambda}\right)_{\lambda \geq 0}} \varphi\right], \quad \operatorname{Re} z \geq 0 .
$$

\section{A relationship between an infinite dimensional Fourier-Mehler transform and the Lévy Laplacian}

For $\sigma, \lambda \geq 0 p>0$ and $N \in \mathbf{N}$ we define spaces $\mathbf{D}_{p ; N}^{\sigma, 0}$ and $\mathbf{D}_{p ; N}^{0, \lambda}$ by

and

$$
\mathbf{D}_{p ; N}^{\sigma, 0}=\left\{\sum_{n=0}^{\infty} \varphi_{n} \in(E)_{p ; \sigma, 0} ; \varphi_{n} \in \mathbf{E}_{\sigma, 0, n}, \quad \sum_{n=0}^{\infty} \alpha_{N}^{\sigma}(n)\left\|\varphi_{n}\right\|_{p ; \sigma, *}^{2}<\infty\right\},
$$

$$
\mathbf{D}_{p ; N}^{0, \lambda}=\left\{\sum_{n=0}^{\infty} \varphi_{n} \in(E)_{p ; 0, \lambda} ; \varphi_{n} \in \mathbf{E}_{0, \sigma, n}, \quad \sum_{n=0}^{\infty} \alpha_{N}^{\lambda}(n)\left\|\varphi_{n}\right\|_{p ; 0, \lambda}^{2}<\infty\right\},
$$

respectively, where $\alpha_{N}^{\tau}(n)=\sum_{\ell=0}^{N}\left(\frac{n \tau^{2}}{|T|}\right)^{2 \ell}, \tau \geq 0$, and

$$
\left\|\varphi_{n}\right\|_{p ; \sigma, *}=\left\|\int_{T^{n}} f_{n}(\mathbf{u}) d\left(\Lambda_{\sigma, 0}\left(u_{1}\right)+\sigma u_{1}\right) \cdots d\left(\Lambda_{\sigma, 0}\left(u_{n}\right)+\sigma u_{n}\right)\right\|_{p ; \sigma, 0},
$$

if $\varphi_{n}=\mathbf{I}_{n}\left(f_{n}\right) \in \mathbf{E}_{\sigma, 0, n}$ for each $n$. These $\mathbf{D}_{p ; N}^{\sigma, 0}$ and $\mathbf{D}_{p ; N}^{0, \lambda}$ are Hilbert spaces with the norms $\|\mid\| \cdot\|\|_{p ; N, \sigma, 0}$ and $\mid\|\cdot\| \|_{p ; N, 0, \lambda}$ given by

$$
\|\varphi\|_{p ; N, \sigma, 0}^{2}=\sum_{n=0}^{\infty} \alpha_{N}^{\sigma}(n)\left\|\varphi_{n}\right\|_{p ; \sigma, *}^{2}, \quad \varphi=\sum_{n=0}^{\infty} \varphi_{n} \in \mathbf{D}_{p ; N}^{\sigma, 0},
$$

and 


$$
\|\varphi\|_{p ; N, 0, \lambda}^{2}=\sum_{n=0}^{\infty} \alpha_{N}^{\lambda}(n)\left\|\varphi_{n}\right\|_{p ; 0, \lambda}^{2}, \quad \varphi=\sum_{n=0}^{\infty} \varphi_{n} \in \mathbf{D}_{p ; N}^{0, \lambda},
$$

respectively. We denote the projective limit space of spaces $\mathbf{D}_{p ; N}^{\sigma, 0}, N \in \mathbf{N}, p>0$, and the projective limit space of spaces $\mathbf{D}_{p ; N}^{0, \lambda}, N \in \mathbf{N}, p>0$, by $\mathbf{D}_{\infty, \infty}^{\sigma, 0}$ and $\mathbf{D}_{\infty, \infty}^{0, \lambda}$, respectively.

A linear operator $K$ defined on $(E)_{\sigma, 0}$ by

$$
K \varphi=\mathcal{U}^{-1}\left[S \varphi\left(e^{i \sigma \xi}\right)\right], \quad \varphi \in(E)_{\sigma, 0},
$$

where $S$ is the $S$-transform on $(E)_{\sigma, 0}^{*}$ given by

$$
S \varphi(\xi)=\exp \left[-\frac{\sigma^{2}}{2} \int_{\mathbf{R}} \xi(t)^{2} d t\right]\left\langle\left\langle\varphi, e^{\langle\cdot, \xi\rangle}\right\rangle\right\rangle_{\sigma, 0}, \quad \xi \in E_{\mathbf{C}} .
$$

A transform $K$ on $\mathbf{D}_{\infty, \infty}^{\sigma, 0}$ is extended to a continuous linear operator from $\mathbf{D}_{p, N}^{\sigma, 0}$ into itself for each $p$ and $N$. We denote the operator by the same notation $K$. The $K$-transform $K \varphi$ of

$$
\varphi=\int_{T^{n}} f\left(u_{1}, \ldots, u_{n}\right) d \Lambda_{\sigma, 0}\left(u_{1}\right) \cdots d \Lambda_{\sigma, 0}\left(u_{n}\right) \in \mathbf{D}_{\infty, \infty}^{\sigma, 0}
$$

is given by

$$
K \varphi=\int_{T^{n}} f\left(u_{1}, \ldots, u_{n}\right) d \Lambda_{0, \sigma}\left(u_{1}\right) \cdots d \Lambda_{0, \sigma}\left(u_{n}\right) \in \mathbf{D}_{\infty, \infty}^{0, \sigma}
$$

for each $p>0, N \in \mathbf{N}$ and $\sigma \geq 0$. Then we have the following.

Theorem 5.1. The operator $K$ gives an isomorphism from $\mathbf{D}_{p, N}^{\sigma, 0}$ onto $\mathbf{D}_{p, N}^{0, \sigma}$ for each $p>0, N \in \mathbf{N}$ and $\sigma>0$.

Proof. It is obvious that $K$ is a bijection from $\mathbf{D}_{p, N}^{\sigma, 0}$ onto $\mathbf{D}_{p, N}^{0, \sigma}$. For any $\varphi=\sum_{n=0}^{\infty} \varphi_{n} \in \mathbf{D}_{p, N}^{\sigma, 0}, \varphi_{n}=$ $\int_{T^{n}} f_{n}\left(u_{1}, \ldots, u_{n}\right) d \Lambda_{\sigma, 0}\left(u_{1}\right) \cdots d \Lambda_{\sigma, 0}\left(u_{n}\right)$, we obtain that

$$
\begin{aligned}
\|K \varphi\| \|_{p ; N, 0, \sigma}^{2}= & \sum_{n=0}^{\infty} \alpha_{N}^{\sigma}(n)\left\|K \varphi_{n}\right\|_{p ; 0, \sigma}^{2} \\
= & \sum_{n=0}^{\infty} \alpha_{N}^{\sigma}(n) \sum_{j=0}^{n}\left(\begin{array}{c}
n \\
j
\end{array}\right)^{2}(n-j) ! \sigma^{2(n-j)} . \\
& \cdot\left|\int_{T^{j}} f_{n}\left(\cdot, \ldots, \cdot, u_{n-j+1}, \ldots, u_{n}\right) d u_{n-j+1} \cdots d u_{n}\right|_{p}^{2} \\
= & \sum_{n=0}^{\infty} \alpha_{N}^{\sigma}(n)\left\|\varphi_{n}\right\|_{p ; \sigma, *}^{2} \\
= & \|\varphi\|_{p ; N, \sigma, 0}^{2},
\end{aligned}
$$

for each $p>0, N \in \mathbf{N}$ and $\sigma>0$. This implies the assertion.

Theorem 5.1 means that the operator $K$ also gives a Gauss-Poisson correspondence. From now on we take $\sigma=1$. Let $\mathcal{N}$ be the number operator on $(E)_{1,0}^{*}$ given by

$$
\mathcal{N} \varphi=\sum_{n=0}^{\infty} n \mathbf{I}_{n}\left(f_{n}\right), \quad \varphi=\sum_{n=0}^{\infty} \mathbf{I}_{n}\left(f_{n}\right) \in(E)_{1,0}^{*} .
$$

The operator $-\mathcal{N}$ is called the Laplace-Beltrami Laplacian. The operator $\mathcal{N}$ is a continuous linear operator from $\mathbf{D}_{p, N+1}^{1,0}$ into $\mathbf{D}_{p, N}^{1,0}$ for every $p>0$ and $N \in \mathbf{N}$ since

$$
\begin{aligned}
\|N \varphi\|_{p ; N, 1,0}^{2}= & \sum_{n=0}^{\infty} \alpha_{N}^{1}(n) \sum_{j=0}^{n}\left(\begin{array}{c}
n \\
j
\end{array}\right)^{2}(n-j) !(n-j)^{2} . \\
& \cdot\left|\int_{T^{j}} f_{n}\left(\cdot, \ldots, \cdot, u_{n-j+1}, \ldots, u_{n}\right) d u_{n-j+1} \cdots d u_{n}\right|_{p}^{2} \\
\leq & |T|^{2} \sum_{n=0}^{\infty} \frac{n^{2}}{|T|^{2}} \alpha_{N}^{1}(n)\left\|\varphi_{n}\right\|_{p ; 1, *}^{2} \\
= & |T|^{2} \sum_{n=0}^{\infty} \sum_{\ell=0}^{N}\left(\frac{n}{|T|}\right)^{2(\ell+1)}\left\|\varphi_{n}\right\|_{p ; 1, *}^{2} \\
\leq & |T|^{2} \sum_{n=0}^{\infty} \alpha_{N+1}^{1}(n)\left\|\varphi_{n}\right\|_{p ; 1, *}^{2}=|T|^{2}\||\varphi|\|_{p ; N+1,1,0}^{2}
\end{aligned}
$$


for any $\varphi=\sum_{n=0}^{\infty} \varphi_{n} \in \mathbf{D}_{p, N+1}^{1,0}$. Since

$$
\Delta_{L} K \varphi_{n}=-\frac{n}{|T|} K \varphi_{n}, \quad \varphi_{n} \in \mathbf{E}_{1,0, n}, \quad n=0,1,2, \ldots,
$$

from Theorem 3.1, we have the following relationship between the Laplace Beltrami Laplacian and the Lévy Laplacian through the operator $K$.

Proposition 5.2. For all $\varphi \in \mathbf{D}_{\infty, \infty}^{1,0}$ we have

$$
K[-\mathcal{N} \varphi]=|T| \Delta_{L} K[\varphi] .
$$

Remark 5.3. Using the white noise differentiation $\partial_{t}$ on $\mathbf{D}_{\infty, \infty}^{1,0}$, we can define an operator $\partial_{K, t}$ on $\mathbf{D}_{\infty, \infty}^{0,1}$ by $\partial_{K, t}=K \partial_{t} K^{-1}$. We also define an operator $\mathcal{N}_{K}$ by $\mathcal{N}_{K}=\int_{\mathbf{R}} \partial_{K, t}^{*} \partial_{K, t} d t$. Then we obtain that

$$
\begin{aligned}
\mathcal{N}_{K} & =\int_{\mathbf{R}} \partial_{K, t}^{*} \partial_{K, t} d t \\
& =\int_{\mathbf{R}}\left(K^{-1}\right)^{*} \partial_{t}^{*} K^{*} K \partial_{t} K^{-1} d t \\
& =\int_{\mathbf{R}} K \partial_{t}^{*} \partial_{t} K^{-1} d t=K \mathcal{N} K^{-1} .
\end{aligned}
$$

This means that $-|T| \Delta_{L}$ acts as the number operator on $\mathbf{D}_{\infty, \infty}^{0,1}$ by $\partial_{K, t}$.

Moreover, by Proposition 5.2, we have a relationship between the semigroup generated by the Laplace-Beltrami Laplacian and that generated by the Lévy Laplacian as follows.

Theorem 5.4. For all $\varphi \in \mathbf{D}_{\infty, \infty}^{1,0}$ we have

$$
K\left[e^{\left.-\frac{t}{\left.T T\right|^{\mathcal{N}}} \varphi\right]}=G_{t}^{1} K[\varphi]\right.
$$

where $G_{t}^{1} \equiv e^{t \Delta_{L}}$.

The operator $K$ also implies an interesting relationship between an infinite dimensional Fourier-Mehler transform and the one-parameter group generated by the Lévy Laplacian. The operator $K$ can be extended to a linear operator defined on a domain $\mathscr{D}$ consisting of white noise distributions $\Phi$ in $(E)_{1,0}^{*}$ such that $S \Phi\left(e^{i \xi}\right)$ exists in $\mathcal{U}\left[(E)_{0,1}^{*}\right]$ since the $\mathcal{U}$-transform is defined on $(E)_{0,1}^{*}$. We denote this extension by the same notation $K$. The operator is a bijection from $\mathscr{D}$ onto $K[D D]$.

For $\sigma, \lambda \geq 0, p>0$ and $N \in \mathbf{N}$, we define the sapces $\mathbf{D}_{-p ; N}^{\sigma, 0}$ and $\mathbf{D}_{-p ; N}^{0, \lambda}$ as the completions of $\mathbf{D}_{\infty, \infty}^{\sigma, 0}$ and $\mathbf{D}_{\infty, \infty}^{0, \lambda}$ by the norms ||$|\cdot|||_{-p ; N, \sigma, 0}$ and $\left|\left\|\cdot|\||_{-p ; N, 0, \lambda}\right.\right.$ given by

$$
\|\varphi\|_{-p ; N, \sigma, 0}^{2}=\sum_{n=0}^{\infty} \alpha_{N}^{\sigma}(n)\left\|\varphi_{n}\right\|_{-p ; \sigma, *}^{2}, \quad \varphi=\sum_{n=0}^{\infty} \varphi_{n} \in \mathbf{D}_{\infty, \infty}^{\sigma, 0}
$$

and

$$
\|\varphi\|_{-p ; N, 0, \lambda}^{2}=\sum_{n=0}^{\infty} \alpha_{N}^{\lambda}(n)\left\|\varphi_{n}\right\|_{-p ; 0, \lambda}^{2}, \quad \varphi=\sum_{n=0}^{\infty} \varphi_{n} \in \mathbf{D}_{\infty, \infty}^{0, \lambda},
$$

respectively. Then the operator $K$ becomes an isomorphism from $\mathbf{D}_{-p ; N}^{\sigma, 0}$ onto $\mathbf{D}_{-p ; N}^{0, \sigma}$.

The Kuo's Fourier-Mehler transform $\widetilde{F}_{\theta}, \theta \in \mathbf{R}$, depending on $T$ is defined by

$$
S\left[\mathcal{F}_{\theta} \varphi\right](\xi)=S \varphi\left(e^{i \theta} \xi\right) \exp \left[\frac{i}{2} e^{i \theta} \sin \theta \int_{T} \xi(t)^{2} d t\right], \quad \xi \in E_{\mathbf{C}} .
$$

This transform is an infinite dimensional Fourier-Mehler transform on $(E)_{1,0}^{*}$ and has many interesting properties in the white noise theory. (See [15]) Let $g$ be a Gaussian white noise distribution in $(E)_{1,0}^{*}$ given by

$$
S g(\xi)=\exp \left[\frac{1}{4} \int_{T} \xi(t)^{2} d t\right], \quad \xi \in E_{\mathbf{C}} .
$$

By Theorem 5.4, we see that

$$
G_{-i \theta|T|}^{1} K \varphi=K\left[e^{i \theta N} \varphi\right], \quad \varphi \in \mathbf{D}_{\infty, \infty}^{1,0}
$$

for all $\theta \in \mathbf{R}$. Since

$$
S\left[e^{i \theta \mathcal{N}} g\right](\xi)=\exp \left[\frac{1}{4} e^{2 i \theta} \int_{T} \xi(t)^{2} d t\right]
$$

and 


$$
S\left[e^{-i \frac{\pi}{2} \cdot \mathcal{N}} g\right](\xi)=\exp \left[-\frac{1}{4} \int_{T} \xi(t)^{2} d t\right]
$$

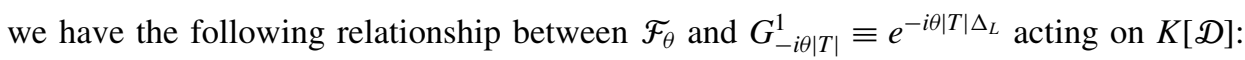

Proposition 5.5. For all $\varphi \in \mathbf{D}_{\infty, \infty}^{1,0}$, we have

$$
K\left[\mathcal{F}_{\theta} \varphi\right]=G_{-i \theta|T|}^{1} K \varphi \diamond G_{-i \frac{\theta}{2}|T|}^{1} K g \diamond G_{-i \frac{\pi}{4}|T|}^{1} K g,
$$

where $\diamond$ means the Wick product defined by

$$
\Phi \diamond \Psi=\mathcal{U}^{-1}[u \Phi \cdot U \Psi], \quad \Phi, \Psi \in K\left[(E)_{0,1}^{*}\right] .
$$

Proof. The $U$-transform $\mathcal{U}[K g]$ of $K g$ is given by

$$
u[K g](\xi)=\exp \left[\frac{1}{4} \int_{T} e^{2 i \xi(u)} d u\right], \quad \xi \in E .
$$

By direct calculations we check that

$$
u\left[G_{-i \frac{\theta}{2}|T|}^{1} K g\right](\xi)=\exp \left[\frac{1}{4} e^{2 i \theta} \int_{T} e^{2 i \xi(u)} d u\right], \quad \xi \in E .
$$

Therefore we have

$$
u\left[G_{-i \frac{\pi}{4}|T|}^{1} K g\right](\xi)=\exp \left[-\frac{1}{4} \int_{T} e^{2 i \xi(u)} d u\right], \quad \xi \in E .
$$

Since $U\left[G_{-i \theta|T|}^{1} K \varphi\right](\xi)=S \varphi\left(e^{i \theta} e^{i \xi}\right), \xi \in E$, we obtain the assertion.

Theorem 4.3 and Proposition 5.5 imply a stochastic expression of $K\left[\mathcal{F}_{\theta} \varphi\right]$. Moreover, since $\Delta_{L}$ is a derivation with respect to the Wick product, we have the following.

Theorem 5.6. For all $\varphi \in \mathbf{D}_{\infty, \infty}^{1,0}, K\left[\mathcal{F}_{\theta} \varphi\right]$ satisfies the equation:

$$
\frac{\partial K\left[\mathcal{F}_{\theta} \varphi\right]}{\partial \theta}=-i|T| \Delta_{L} K\left[\mathcal{F}_{\theta} \varphi\right]+i\left(1-\frac{1}{2} e^{2 i \theta}\right) K\left[\int_{T}: x(t)^{2}: d t\right] \diamond K\left[\mathcal{F}_{\theta} \varphi\right]
$$

where $\int_{T}: x(t)^{2}: d t$ is a Gaussian noise distribution of which the $S$-transform is given by $S\left[\int_{T}: x(t)^{2}: d t\right](\xi)=$ $\int_{T} \xi(t)^{2} d t, \xi \in E_{\mathbf{C}}$.

Proof. We can check that

$$
\begin{aligned}
\frac{\partial K\left[\mathcal{F}_{\theta} \varphi\right]}{\partial \theta}= & \left(-i|T| \Delta_{L} G_{-i \theta|T|}^{1} K \varphi\right) \diamond G_{-i \frac{\theta}{\mid}|T|}^{1} K g \diamond G_{-i \frac{\pi}{4}|T|}^{1} K g \\
& +G_{-i \theta|T|}^{1} K \varphi \diamond\left(-i \frac{1}{2}|T| \Delta_{L} G_{-i \frac{\theta}{2}|T|}^{1} K g\right) \diamond G_{-i \frac{\pi}{4}|T|}^{1} K g
\end{aligned}
$$

and

$$
\begin{aligned}
-i|T| \Delta_{L} K\left[\mathcal{F}_{\theta} \varphi\right]= & \left(-i|T| \Delta_{L} G_{-i \theta|T|}^{1} K \varphi\right) \diamond G_{-i \frac{\theta}{2}|T|}^{1} K g \diamond G_{-i \frac{\pi}{4}|T|}^{1} K g \\
& +G_{-i \theta|T|}^{1} K \varphi \diamond\left(-i|T| \Delta_{L} G_{-i \frac{\theta}{2}|T|}^{1} K g\right) \diamond G_{-i \frac{\pi}{4}|T|}^{1} K g \\
& +G_{-i \theta|T|}^{1} K \varphi \diamond G_{-i \frac{\theta}{2}|T|}^{1} K g \diamond\left(-i|T| \Delta_{L} G_{-i \frac{\pi}{4}|T|}^{1} K g\right)
\end{aligned}
$$

since the Lévy Laplacian has the derivation property with respect to the Wick product. Therefore we obtain that

$$
\begin{aligned}
\frac{\partial K\left[\mathcal{F}_{\theta} \varphi\right]}{\partial \theta}= & -i|T| \Delta_{L} K\left[\mathcal{F}_{\theta} \varphi\right] \\
& +G_{-i \theta|T|}^{1} K \varphi \diamond\left(-i \frac{|T|}{2} \Delta_{L} G_{-i \frac{\theta}{2}|T|}^{1} K g\right) \diamond G_{-i \frac{\pi}{4}|T|}^{1} K g \\
& +G_{-i \theta|T|}^{1} K \varphi \diamond G_{-i \frac{\theta}{2}|T|}^{1} K g \diamond\left(-i|T| \Delta_{L} G_{-i \frac{\pi}{4}|T|}^{1} K g\right) .
\end{aligned}
$$

Since $U\left[\Delta_{L} G_{-i \frac{\theta}{2}|T|}^{1} K g\right](\xi)=-e^{2 i \theta} \int_{T} e^{2 i \xi(u)} d u \exp \left[\frac{1}{4} e^{2 i \theta} \int_{T} e^{2 i \xi(u)} d u\right]$, we have the assertion.

Remark 5.7. The equation in Theorem 5.6 implies 


$$
\begin{aligned}
\left.\frac{\partial K\left[\mathcal{F}_{\theta} \varphi\right]}{\partial \theta}\right|_{\theta=0} & =-i|T| \Delta_{L} K \varphi+\frac{i}{2} K\left[\Delta_{G}^{*}\right] K \varphi \\
& =K\left[i\left(\mathcal{N}+\frac{1}{2} \Delta_{G}^{*}\right) \varphi\right],
\end{aligned}
$$

where $\Delta_{G}^{*}$ is the adjoint operator of the Gross Laplacian $\Delta_{G}$. (See [22])

\section{Acknowledgements}

The author would like to express his deep thanks to the organizers, Professors N. Obata, F. Hiai (Tohoku University), U. C. Ji (Chungbuk National University) and M. Bozejko (Wroclaw University) for the inivitation to the stimulating Workshop "the 8th Sendai Workshop on Infinite Dimensional Analysis and Quantum Probability," Tohoku University, November 10-12, 2008. This work was supported by the KOSEF-JSPS Joint Reseach Project (2007-2009) "Noncommutative Stochastic Analysis and its Applications to Network Science" and was also supported, in part, by Grant-in-Aid Scientific Research 21540151. The author is grateful for their supports.

\section{REFERENCES}

[1] Accardi, L., and Bogachëv, V., "The Ornstein-Uhlenbeck process associated with the Lévy Laplacian and its Dirichlet form," Prob. Math. Stat., 17: 95-114 (1997).

[2] Accardi, L., Gibilisco, P., and Volovich, I. V., "Yang-Mills gauge fields as harmonic functions for the Lévy Laplacian," Russ. J. Math. Phys., 2: 235-250 (1994).

[3] Accardi, L., and Smolyanov, O. G., Trace formulae for Levy-Gaussian measures and their application, in "Mathematical Approach to Fluctuations Vol. II (Hida, T., Ed.),” World Scientific, pp. 31-47 (1995).

[4] Accardi, L., Barhoumi, A., and Ouerdiane, H., "A quantum approach to Laplace operators," Infin. Dimen. Anal. Quantum Probab. Rel. Top., 9: 215-248 (2006).

[5] Chung, D. M., and Ji, U. C., "Transformation groups on white noise functionals and their applications," Appl. Math. Optim., 37: 205-223 (1998).

[6] Chung, D. M., Ji, U. C., and Saitô, K., "Cauchy problems associated with the Lévy Laplacian in white noise analysis," Infin. Dimen. Anal. Quantum Probab. Rel. Top., 2: 131-153 (1999).

[7] Feller, M. N., "Infinite-dimensional elliptic equations and operators of Lévy type," Russ. Math. Surveys, 41: 119-170 (1986).

[8] Hida, T., "Analysis of Brownian Functionals," Carleton Math. Lect. Notes, No. 13, Carleton University, Ottawa (1975).

[9] Hida, T., Kuo, H.-H., and Obata, N., "Transformations for white noise functionals," J. Funct. Anal., 111: $259-277$ (1993).

[10] Hida, T., Kuo, H.-H., Potthoff, J., and Streit, L., "White Noise: An Infinite Dimensional Calculus,” Kluwer Academic (1993).

[11] Ishikawa, A., and Saitô, K., “The Lévy Laplacian and the Lévy Process,” RIMS Kokyuroku, 1507: 14-25 (2006).

[12] Ji, U. C., and Saitô, K., "A similarity between the Gross Laplacian and the Lévy Laplacian," Infin. Dimen. Anal. Quantum Probab. Rel. Top., 10: 261-276 (2007).

[13] Kubo, I., and Takenaka, S., "Calculus on Gaussian white noise I-IV," Proc. Japan Acad., 56A: 376-380 (1980); 56A: 411-416 (1980); 57A: 433-436 (1981); 58A: 186-189 (1982).

[14] Kuo, H.-H., "Fourier-Mehler transforms of generalized Brownian functionals," Proc. Japan Acad., 59A: 312-314 (1983).

[15] Kuo, H.-H., "White Noise Distribution Theory," CRC Press (1996).

[16] Kuo, H.-H., Obata, N., and Saitô, K., "Lévy Laplacian of generalized functions on a nuclear space,” J. Funct. Anal., 94: 74-92 (1990).

[17] Kuo, H.-H., Obata, N., and Saitô, K., "Diagonalization of the Lévy Laplacian and related stable processes," Infin. Dimen. Anal. Quantum Probab. Rel. Top., 5: 317-331 (2002).

[18] Lévy, P., "Leçons d'Analyse Fonctionnelle," Gauthier-Villars, Paris (1922).

[19] Léandre, R., and Volovich, I. A., "The stochastic Lévy Laplacian and Yang-Mills equation on manifolds," Infin. Dimen. Anal. Quantum Probab. Rel. Top., 4: 161-172 (2001).

[20] Nishi, K., and Saitô, K., An infinite dimensional stochastic process and the Lévy Laplacian acting on WND-valued functions, in "Quantum Inofrmation and Complexity (Hida, T., Ed.)," pp. 376-390, World Scientific (2004).

[21] Obata, N., "A characterization of the Lévy Laplacian in terms of infinite dimensional rotation groups," Nagoya Math. J., 118: 111-132 (1990).

[22] Obata, N., "White Noise Calculus and Fock Space," Lect. Notes in Math. Vol. 1577, Springer-Verlag (1994).

[23] Obata, N., "Quadratic quantum white noises and Lévy Laplacian," Nonlinear Analysis, 47: 2437-2448 (2001).

[24] Obata, N., and Saitô, K., Cauchy processes and the Lévy Laplacian, in "Quantum Probability and White Noise Analysis 16 (Obata, N., Matsui, T., and Hora, A., Eds.),” pp. 360-373, World Scientific (2002).

[25] Saitô, K., "Itô’s formula and Lévy’s Laplacian I,” Nagoya Math. J., 108: 67-76 (1987); “II,” ibid., 123: 153-169 (1991).

[26] Saitô, K., “A $\left(C_{0}\right)$-group generated by the Lévy Laplacian II,” Infin. Dimen. Anal. Quantum Probab. Rel. Top., 1: 425-437 (1998).

[27] Saitô, K., “A stochastic process generated by the Lévy Laplacian,” Acta Appl. Math., 63: 363-373 (2000).

[28] Saitô, K., An infinite dimensional Laplacian acting on multiple Wiener integrals by some Lévy processes, in "Infinite Dimensional Harmonic Analysis III (Heyer, H., Hirai, T., Kawazoe, T., and Saitô, K., Eds.), pp. 265-276, World Scientific (2005).

[29] Saitô, K., and Sakabe, K., An infinite dimensional Fourier-Mehler transform and the Lévy Laplacian, in "Quantum Probability and White Noise Analysis 22 (Sengupta, A. N., and Sundar, P., Eds.)," pp. 149-160, World Scientific (2008). 
[30] Saitô, K., and Tsoi, A. H., The Lévy Laplacian as a self-adjoint operator, in "Quantum Information (Hida, T., and Saitô, K., Eds.)," pp. 159-171, World Scientific (1999).

[31] Saitô, K., and Tsoi, A. H., "The Lévy Laplacian acting on Poisson noise functionals," Infin. Dimen. Anal. Quantum Probab. Rel. Top., 2: 503-510 (1999). 